\title{
ANÁLISE DE ASPECTOS DA DINÂMICA E CONTROLE DE UM REATOR COM MEMBRANA PARA DESIDROGENAÇÃO DO ETILBENZENO
}

\author{
F. A. COELHO, G. POSSANI e R. J. ZEMP \\ Universidade Estadual de Campinas, Faculdade de Engenharia Química \\ E-mail para contato: filipea_c@ @otmail.com
}

RESUMO - A demanda crescente por processos mais eficientes e menos poluentes é a força motriz da intensificação de processos. Nesta área, destacam-se o uso de equipamentos multifuncionais, como os reatores com membrana que integram em um único equipamento uma unidade de reação química e uma unidade de separação. Dado o acoplamento das variáveis de operação, a análise da dinâmica e controle desses equipamentos é fundamental para estabelecer condições operacionais adequadas. Assim, neste trabalho foi desenvolvido o modelo dinâmico de um reator de desidrogenação de etilbenzeno com membrana composta de paládio. Foram feitas análises qualitativas do comportamento dinâmico diante de perturbações nas variáveis de processo e também foi utilizada uma estratégia baseada na matriz de ganhos relativos para estabelecer a influência das principais variáveis do equipamento (temperatura e vazão do gás de arraste, razão vapor/etilbenzeno na alimentação e pressão do reator) na conversão e rendimento da reação.

\section{INTRODUÇÃO}

O desenvolvimento de processos químicos mais eficientes, com o intuito de promover mudanças nos modelos atuais de produção e processamento, que proporcionem tecnologias mais limpas, menores e energeticamente mais eficientes constitui a Intensificação de Processos de acordo com Stankiewicz e Moulijn (2000). Um tipo de equipamento que contribui nesta área são os chamados reatores com membrana, que além de promoverem a separação de uma mistura de substâncias, também podem, simultaneamente, conduzir uma reação química, neste caso também conhecidos como reatores multifuncionais, conforme Kumar et al. (2006). Um exemplo para o uso nesses reatores é a desidrogenação catalítica do etilbenzeno para a produção de estireno (Quicker et al., 2000).

Na desidrogenação catalítica do etilbenzeno, que corresponde a $90 \%$ da produção de estireno de acordo com Abo-Gander et al. (2008), o equilíbrio termodinâmico limita a conversão do etilbenzeno em valores próximos a 50\%, porém se o hidrogênio, que é um subproduto, for removido do meio reacional haverá um aumento da conversão do etilbenzeno em estireno, além de aumentar a seletividade na produção de estireno. Esta remoção pode ser feita utilizando-se os reatores com membranas compostas de paládio, pois o paládio apresenta uma característica única de ser permeável somente ao hidrogênio (Dittmeyer et al., 2001). 
Outro fator importante referente à Intensificação de Processos envolvendo os reatores com membrana é que, de acordo com Strathmann (2001), membranas adequadas são a chave em certas aplicações, porém elas são apenas uma pequena parte de todos os equipamentos envolvidos no processo, sendo que em muitos casos seus custos são irrelevantes quando comparados com os componentes periféricos. Nesse contexto é necessário se conhecer as condições operacionais destes processos envolvendo membranas e também seu comportamento frente a diferentes perturbações destas condições de operação. Conhecendo essas informações do sistema é possível ter uma melhor estimativa das especificações necessárias dos equipamentos envolvidos para o desenvolvimento do projeto de um sistema com reator com membrana e também da influência das variáveis de operação na conversão e rendimento da reação.

A dinâmica e controle de equipamentos com intensificação ainda não é um assunto muito discutido na literatura e, em especial, para reatores com membrana esse conhecimento é essencial, dado que a incorporação da membrana acopla diversas variáveis do sistema e a dinâmica do equipamento difere significativamente em relação aos reatores convencionais. Um dos poucos trabalhos que aborda o tema da dinâmica em reatores com membrana composta de paládio é o trabalho de Koc et al. (2011) no qual estuda a reação de deslocamento de gás d'água (Water Gas Shift Reaction) - utilizada na produção de hidrogênio, por meio da conversão do monóxido de carbono em $\mathrm{CO}_{2}$ e da água em $\mathrm{H}_{2}$ - conduzida em um reator com membrana composta de $\mathrm{Pd} / \mathrm{Au}$ e $\mathrm{Pd} / \mathrm{Cu}$ a fim de analisar e caracterizar o comportamento transiente deste sistema.

Assim, o objetivo deste trabalho foi analisar o comportamento dinâmico para um sistema de desidrogenação do etilbenzeno em estireno usando uma membrana composta de paládio em suporte de aço inoxidável poroso. Também foi utilizada uma metodologia para pareamento de variáveis manipuladas/controladas para determinar quais variáveis interferem independentemente na conversão e rendimento.

\section{MATRIZ MGR}

A matriz MGR (Matriz de Ganhos Relativos) consiste em uma metodologia sistemática para análise de problemas de controle multivariável. Utilizando a matriz de ganhos em estado estacionário $(K)$, é possível medir interações no processo e extrair informação sobre o melhor pareamento de variáveis controladas e manipuladas (Seborg et al., 2004).

Resumidamente, a matriz MGR é calculada conforme a Equação 1, onde $\otimes$ representa o produto elemento a elemento ou produto de Schur, e a matriz de ganhos $K$ é calculada de acordo com a Equação 2, onde $y$ representa as variáveis controladas e $u$, as variáveis manipuladas.

$$
\begin{gathered}
\Lambda=K \otimes\left(K^{-1}\right)^{T} \\
K_{i j}=\frac{\Delta y_{i}}{\Delta u_{j}}
\end{gathered}
$$

O pareamento é feito analisando-se os elementos $K_{i j}$, que podem assumir os seguintes 
valores: $K_{i j}=1$, não há interação com outras malhas de controle e o par $i-j$ deve ser usado; $K_{i j}=0$, variável manipulada $j$ não afeta a variável controlada $i ; K_{i j}=0,5$, ocorre grande interação com outras malhas de controle; $0,5<K_{i j}<1$, ocorre interação com outras malhas, entretanto, o par $i-j$ pode ser usado visto que minimiza as interações; $K_{i j}>1$, a interação com outras malhas reduz o efeito da malha de controle e $K_{i j}<0$, o par $i-j$ pode levar a uma operação instável.

Como a matriz MGR só pode ser calculada para uma matriz $K$ quadrada, é necessário computar as várias combinações possíveis de variáveis manipuladas/controladas até encontrar a que apresente o melhor resultado. Neste trabalho as variáveis que serão avaliadas no pareamento são: vazão de alimentação do retentato, vazão do gás de arraste, temperatura da alimentação do retentato, temperatura de alimentação do gás de arraste e razão vapor/etilbenzeno na alimentação.

\section{EQUACIONAMENTO DO SISTEMA}

O esquema do reator está ilustrado na Figura 1. O tubo interno, que neste trabalho considera-se onde ocorre a reação, é chamado de retentato e apenas o hidrogênio liberado na reação atravessa o filme de paládio até o outro lado do reator, chamado de permeado. Neste lado passa um gás de arraste, que neste trabalho foi considerado nitrogênio. $\mathrm{O}$ modelo utilizado despreza a queda de pressão em ambos os lados do equipamento, por ser muito pequena.

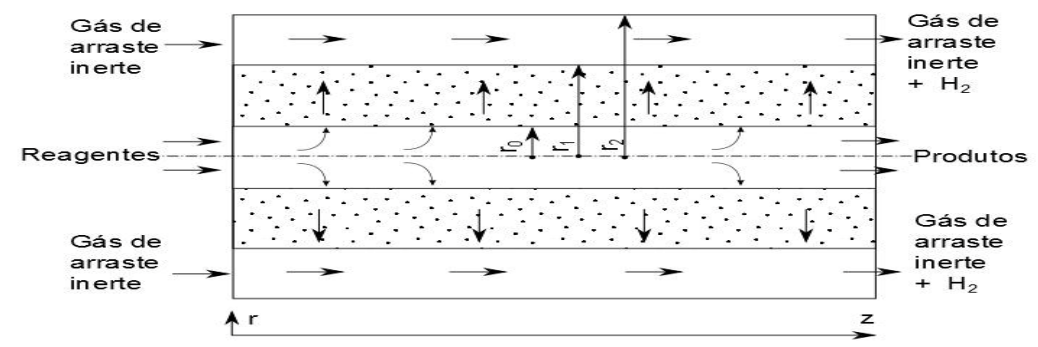

Figura 1. Ilustração do sistema reator + membrana.

Os balanços de massa e energia para ambos os lados, assim como a equação que descreve a permeação do hidrogênio (Lei de Sieverts, na Equação 6) são apresentados nas Equações 3 até 8.

$$
\begin{gathered}
\frac{\partial c_{i}^{\mathrm{R}}}{\partial t}=-v^{\mathrm{R}} \frac{\partial c_{i}^{\mathrm{R}}}{\partial z}+(1-\varepsilon) \rho_{b} \sum_{j}\left(v_{j, i} r_{j}^{\prime}\right) \\
\frac{\partial c_{\mathrm{H}_{2}}^{\mathrm{R}}}{\partial t}=-v^{\mathrm{R}} \frac{\partial c_{\mathrm{H}_{2}}^{\mathrm{R}}}{\partial z}+(1-\varepsilon) \rho_{b} \sum_{j}\left(v_{j, \mathrm{H}_{2}} r_{j}^{\prime}\right)-\frac{2}{r_{1}} J_{\mathrm{H}_{2}} \\
\frac{\partial c_{\mathrm{H}_{2}}^{\mathrm{P}}}{\partial t}=-v^{\mathrm{P}} \frac{\partial c_{\mathrm{H}_{2}}^{\mathrm{P}}}{\partial z}+\frac{2 r_{1}}{r_{2}^{2}-r_{1}^{2}} J_{\mathrm{H}_{2}} \\
J_{\mathrm{H}_{2}}=\frac{2,3 \cdot 10^{-7} \exp (-21700 / R T) \cdot 3,03 \cdot 10^{2} T^{-1,0358}}{\ln \left(r_{1} / r_{0}\right) \sqrt{P_{0}}\left(r_{1}-r_{0}\right)}\left(\sqrt{P_{\mathrm{H}_{2}}^{\mathrm{R}}}-\sqrt{P_{\mathrm{H}_{2}}^{\mathrm{P}}}\right)
\end{gathered}
$$




$$
\begin{gathered}
\frac{\partial T^{\mathrm{R}}}{\partial t}=-v^{\mathrm{R}} \frac{\partial T^{\mathrm{R}}}{\partial z}+\frac{(1-\varepsilon) \rho_{b} \sum_{j}\left(-\Delta H_{r, j} r_{j}^{\prime}\right)}{\rho^{\mathrm{R}} c_{p}^{\mathrm{R}}}+\frac{2 U_{1}}{\rho^{\mathrm{R}} c_{p}^{\mathrm{R}} r_{1}}\left(T^{\mathrm{P}}-T^{\mathrm{R}}\right) \\
\frac{\partial T^{\mathrm{P}}}{\partial t}=-v^{\mathrm{P}} \frac{\partial T^{\mathrm{P}}}{\partial z}-\frac{2 U_{1}}{\rho^{\mathrm{R}} c_{p}^{\mathrm{R}} r_{1}}\left(T^{\mathrm{P}}-T^{\mathrm{R}}\right)
\end{gathered}
$$

Onde, $c$ é a concentração molar $\left[\mathrm{mol} / \mathrm{m}^{3}\right] ; t$ é o tempo $[\mathrm{s}] ; v$ é a velocidade $[\mathrm{m} / \mathrm{s}] ; z$ é a direção axial [m]; $\varepsilon$ é a fração de vazios do leito; $\rho_{b}$ é densidade do catalisador $\left[\mathrm{kgcat} / \mathrm{m}^{3}\right] ; v$ é o coeficiente estequiométrico; $r$ 'é a taxa de reação $\left[\mathrm{kmol} \cdot \mathrm{kgcat}^{-1} \mathrm{~s}^{-1}\right] ; r_{1}, r_{2}$ e $r_{3}$ são os raios $[\mathrm{m}] ; J$ é a taxa de transferência de massa através da membrana $[\mathrm{mol} / \mathrm{s}] ; P$ é a pressão total $[\mathrm{Pa}] ; P_{\mathrm{H} 2}$ é a pressão parcial do $\mathrm{H}_{2}[\mathrm{~Pa}] ; P_{0}$ é a pressão de referência de $1 \mathrm{~atm} ; T$ é a temperatura $[\mathrm{K}] ; \Delta H_{r}$ é a entalpia de reação $[\mathrm{kJ} / \mathrm{kmol}] ; U$ é coeficiente global de transferência de calor $\left[\mathrm{kJ} \cdot \mathrm{m}^{-2} \mathrm{~K}^{-1}\right] ; \rho$ é a densidade do fluido $\left[\mathrm{mol} / \mathrm{m}^{3}\right] ; c_{p}$ é a capacidade calorífica $\left[\mathrm{kJ} \cdot \mathrm{mol}^{-1} \mathrm{~K}^{-1}\right.$; Ré a constante dos gases $\left[\mathrm{J} \cdot \mathrm{mol}^{-1} \mathrm{~K}^{-1}\right]$; R é o sobrescrito para o lado do retentato e P é o sobrescrito para lado do permeado.

As reações e as respectivas taxas estão apresentadas na Tabela 1. As entalpias de reação podem ser encontradas em Hermann et al. (1997). O $U$ foi calculado considerando-se o coeficiente convectivo para o lado do retentato através do modelo de Calderbank e Pogorski (1957) na Equação 9, modelo utilizado para leitos empacotados, e para o lado do permeado, o modelo de Hausen (1959) na Equação 10, recomendado para escoamento laminar em tubos horizontais (inclusive ânulos).

\begin{tabular}{|c|c|}
\hline Reação & Taxa $/ \mathrm{kmol} \cdot \mathrm{kg}_{\mathrm{cat}}{ }^{-1} \mathrm{~s}^{-1}$ \\
\hline $\mathrm{EB} \leftrightarrow \mathrm{ST}+\mathrm{H}_{2}$ & $r_{1}=5,95 \cdot 10^{(-4770 / T+4,1)}\left[P_{\mathrm{EB}}-P_{\mathrm{ST}} P_{\mathrm{H}_{2}} / \mathrm{K}_{\mathrm{P}}\right]$ \\
\hline $\mathrm{EB} \rightarrow \mathrm{B}+\mathrm{C}_{2} \mathrm{H}_{4}$ & $r_{2}=1,03 \cdot 10^{1} \exp (-208000 / R T) P_{\mathrm{EB}}$ \\
\hline $\mathrm{EB}+\mathrm{H}_{2} \rightarrow \mathrm{T}+\mathrm{CH}_{4}$ & $r_{3}=8,52 \cdot 10^{-10} \exp (-91500 / R T) P_{\mathrm{EB}} P_{\mathrm{H}_{2}}$ \\
\hline $\mathrm{H}_{2} \mathrm{O}+0,5 \mathrm{C}_{2} \mathrm{H}_{4} \rightarrow \mathrm{CO}+2 \mathrm{H}_{2}$ & $r_{4}=5,72 \cdot 10^{-8} \exp (-104000 / R T) P_{\mathrm{H}_{2} \mathrm{O}} \sqrt{P_{\mathrm{C}_{2} \mathrm{H}_{4}}}$ \\
\hline $\mathrm{H}_{2} \mathrm{O}+\mathrm{CH}_{4} \rightarrow \mathrm{CO}+3 \mathrm{H}_{2}$ & $r_{5}=1,04 \cdot 10^{-11} \exp (-65700 / R T) P_{\mathrm{H}_{2} \mathrm{O}} P_{\mathrm{CH}_{4}}$ \\
\hline $\mathrm{H}_{2} \mathrm{O}+\mathrm{CO} \rightarrow \mathrm{CO}_{2}+\mathrm{H}_{2}$ & $r_{6}=3,22 \cdot 10^{-6} \exp (-73600 / R T) P_{\mathrm{H}_{2} \mathrm{O}} P_{\mathrm{CO}} P / T$ \\
\hline \multicolumn{2}{|c|}{$\begin{array}{l}\text { EB }=\text { Etilbenzeno, } \mathrm{ST}=\text { Estireno, } \mathrm{B}=\text { Benzeno, } \mathrm{T}=\text { Tolueno } \\
K_{\mathrm{p}}=\exp \left(-122,7 \cdot 10^{3}-126,3 T-2194 \cdot 10^{-3} T^{2} / R T\right) \cdot 10^{5}[\mathrm{~Pa}], 700 \mathrm{~K}<T<980 \mathrm{~K} \\
\qquad h^{\mathrm{R}}=\frac{\lambda}{d_{p}} 3,6\left(\frac{d_{p} G}{\eta \varepsilon_{b}}\right)^{0,365}\end{array}$} \\
\hline
\end{tabular}

Tabela 1 - Sistema reacional no lado do retentato. Adaptado de Hermann et al. (1997). 


$$
h^{\mathrm{P}}=3,66+\frac{0,19 R e^{0,8}}{1+0,117 G z}
$$

Onde $h$ é o coeficiente convectivo $\left[\mathrm{kJ} \cdot \mathrm{m}^{-2} \mathrm{~K}^{-1}\right] ; d_{p}$ é o diâmetro do catalisador $[\mathrm{m}] ; G$ é a velocidade mássica superficial $\left[\mathrm{kg} \cdot \mathrm{m}^{-2} \mathrm{~s}^{-1}\right] ; \eta$ é a viscosidade dinâmica $[\mathrm{Pa} \cdot \mathrm{s}] ;$ Re é o número de Reynolds e $G z$ é o número de Graetz.

\section{RESULTADOS}

O modelo foi discretizado por diferenças finitas e implementado em Matlab Simulink ${ }^{\circledR}$, onde foi utilizado o solver ode15s, visto que o sistema apresenta rigidez. Dada a geometria do equipamento e as condições operacionais normais na Tabela 2 , a conversão é de aproximadamente $86 \%$ e o rendimento de $82 \%$.

Tabela 2 - Geometria do equipamento e condições operacionais.

\begin{tabular}{|c|c|}
\hline Dados & Valor \\
\hline Comprimento & $0,5 \mathrm{~m}$ \\
\hline Raio total do equipamento $\left(r_{2}\right)$ & $0,0254 \mathrm{~m}$ \\
\hline Espessura da membrana $\left(r_{1}-r_{0}\right)$ & $20 \mu \mathrm{m}$ \\
\hline Diâmetro hidráulico do permeado $\left(r_{2}-r_{1}\right)$ & $5 \mathrm{~mm}$ \\
\hline Diâmetro do catalisador & $3 \mathrm{~mm}$ \\
\hline Densidade do catalisador & $2150 \mathrm{~kg} / \mathrm{m}^{3}$ \\
\hline Fração de vazios do leito & 0,5 \\
\hline Temperatura de alimentação do retentato & $893 \mathrm{~K}$ \\
\hline Temperatura de alimentação do gás de arraste & $893 \mathrm{~K}$ \\
\hline Pressão no retentato & $250 \mathrm{kPa}$ \\
\hline Pressão no permeado & $110 \mathrm{kPa}$ \\
\hline Razão vapor/etilbenzeno & 12 \\
\hline Razão gás de arraste/etilbenzeno & 200 \\
\hline WHSV & $1 \mathrm{~h}^{-1}$ \\
\hline
\end{tabular}

Os resultados com a matriz MGR mostraram que os melhores pares de variáveis manipuladas/controladas são vazão de alimentação do retentato/conversão e temperatura da alimentação do retentato/rendimento. É interessante observar que as variáveis mais adequadas para o controle da conversão e rendimento ficam no lado do retentato (lado da reação) e variáveis relacionadas ao gás de arraste, que é o diferencial do reator com membrana contra o reator convencional, interferem simultaneamente na conversão e rendimento, sendo inadequadas ao controle do equipamento.

Com uma análise de sensibilidade verificou-se que uma variação de até $\pm 50 \%$ na vazão de alimentação em relação ao ponto de operação não interfere mais que $0,3 \%$ no rendimento. Assumindo que o equipamento apresenta rendimento acima de $70 \%$ (situação verdadeira para a faixa de $\pm 50 \% \mathrm{em}$ 
torno do ponto de operação de todas as variáveis avaliadas neste trabalho, exceto as temperaturas das alimentações), pode-se reduzir o controle multivariável da conversão e rendimento para apenas o controle da conversão utilizando a vazão de alimentação no retentato como variável manipulada.

Na prática, dificilmente a conversão seria utilizada diretamente como variável controlada, em função da dificuldade de se medir a composição da saída.Tentativas anteriores dos presentes autores revelaram a dificuldade de utilizar outra variável manipulada em um controle SISO (Single Input Single Output) a fim de manter a conversão e essa dificuldade emerge da complexidade da operação do equipamento e das diversas fontes de perturbações que podem interferir em seu funcionamento. Assim, chega-se a conclusão que o controle do equipamento pode ser feito de duas maneiras: monovariável utilizando a conversão como variável controlada ou multivariável com diversas malhas de controle mantendo todas as variáveis que interferem no sistema no set-point para garantir a conversão.

A partir desse ponto de vista, um controle monovariável onde seja possível inferir a conversão é desejável pela sua simplicidade. A inferência da composição de saída, e portanto, da conversão, pode ser feita na prática por meio de modelos matemáticos baseados em balanços de massa e energia, como o modelo empregado neste trabalho, ou usando modelos empíricos como redes neurais através de um histórico de dados do equipamento e da análise da composição dos produtos.

Dessa maneira, considerando ser possível estimar em tempo real a conversão, a malha de controle foi estabelecida utilizando a vazão de alimentação do retentato como variável manipulada e a conversão como variável controlada. O controlador utilizado foi um controlador PI sintonizado pelo algoritmo padrão do bloco PID do Simulink, cujos parâmetros resultantes são: $K_{p}=-43,667$ e $K_{i}=-78,562$.

Os resultados das Figuras 2 e 3 mostram perturbações de $+30 \%$ e $-30 \%$ nos tempos 100 s e 200 s, respectivamente, na razão vapor/etilbenzeno $(S O)$ e vazão do gás de arraste $\left(F_{\mathrm{p} 0}\right)$ e de $+5 \%$ e $-5 \%$ nas temperaturas de alimentação $\left(T_{\mathrm{r} 0}\right.$ e $\left.T_{\mathrm{p} 0}\right)$ (as percentagens foram mais baixas porque $30 \%$ de perturbação equivale a uma variação instantânea infactível de $270^{\circ} \mathrm{C}$ ). Na Figura 2 a malha estava aberta e na Figura 3 o controlador PI estava ligado.
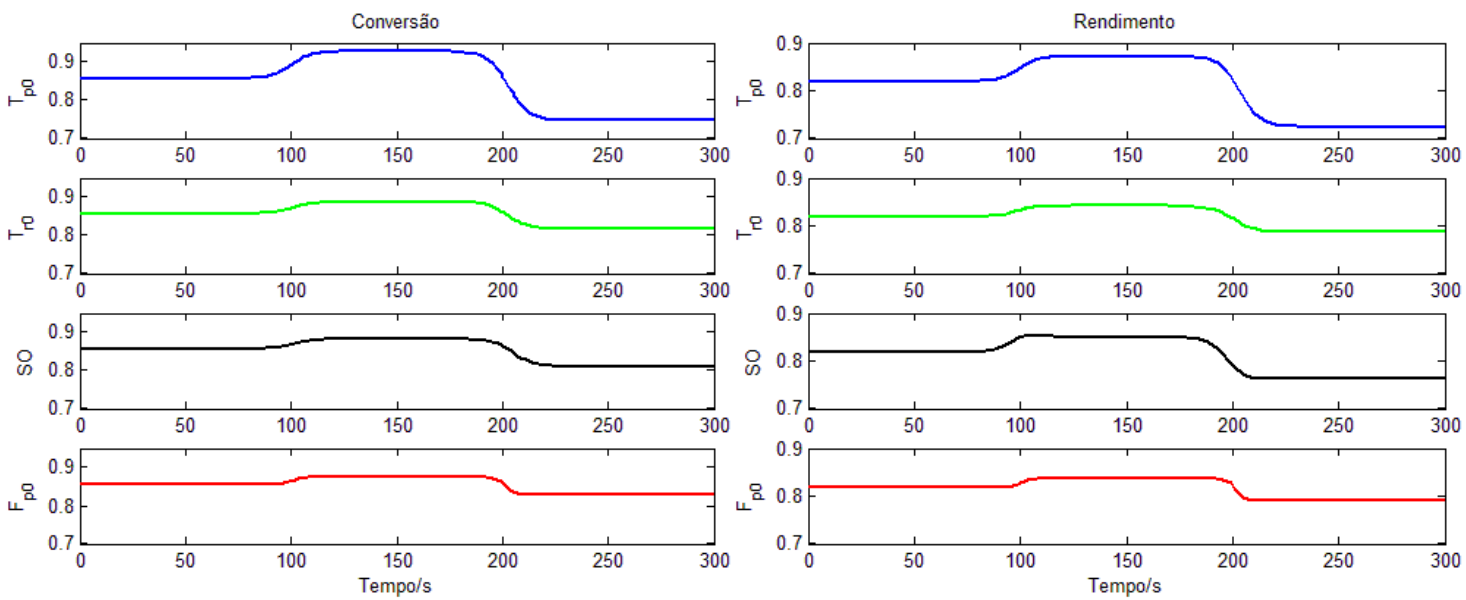

Figura 2 - Resposta da conversão e rendimento (em malha aberta) em função de diferentes perturbações no sistema. 

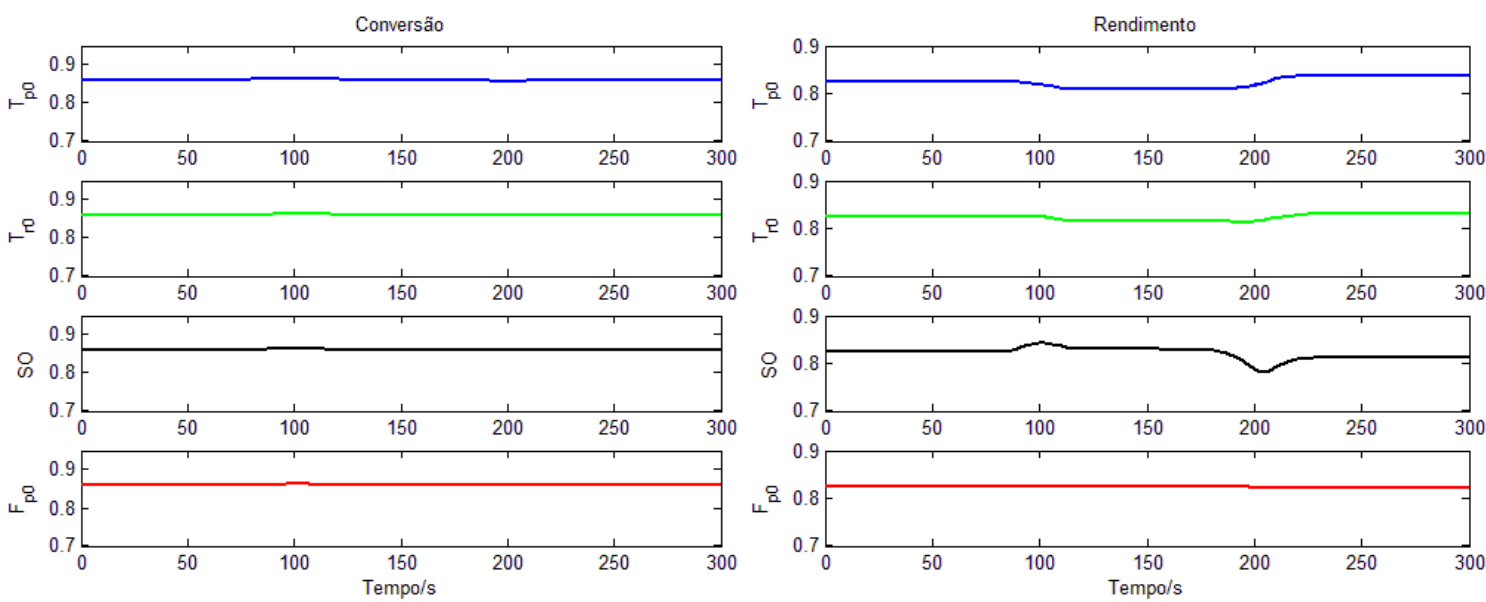

Figura 3-Resposta da conversão e rendimento (com controle regulatório) em função de diferentes perturbações no sistema.

Observa-se que a correção do controlador é razoavelmente robusta em relação a perturbações de diferentes naturezas, mesmo utilizando um simples controlador PI, mostrando o potencial do controle monovariável para manter a conversão do reator. Apesar de não estar sendo controlado, o rendimento também se manteve próximo ao valor de $82 \%$ (valor para as condições operacionais normais) quando a conversão atingia o set-point. O SO foi a variável que apresentou a maior variação na seletividade em malha fechada, mas ainda assim permaneceu acima do valor de $70 \%$, estipulado como aceitável.

\section{CONCLUSÕES}

Neste trabalho foi apresentado o controle de um reator com membrana para desidrogenação de etilbenzeno. Foi demonstrado, através da matriz MGR, que as melhores variáveis para controlar a conversão e rendimento são variáveis típicas de um reator convencional e, portanto, o lado do permeado não possui variáveis que possam ser manipuladas de forma adequada ao controle.

A malha de controle utilizada para controle da conversão utilizou a vazão de alimentação do retentato como variável manipulada em um controlador PI regulatório para manter a conversão no set-point.Verificou-se que o controle se mostrou adequado diante de perturbações diversas do sistema e que apesar do grande acoplamento existente, mediante escolha cuidadosa, é possível manter a conversão nesse tipo de reator sem grandes dificuldades com controle SISO. 


\section{REFERENCIAS}

ABO-GHANDER, N. S.; GRACE, J. R.; ELNASHAIE, S. S. E. H.; LIM C. J., Modeling of a novel membrane reactor to integrate dehydrogenation of ethylbenzene to styrene with hydrogenation of nitrobenzene to aniline, Chemical Engineering Science, v.63, p.1817 - 1826, 2008.

CALDERBANK, P. H.; POGORSKI, L. A. Transactions of the Institution of ChemicalEngineers, v.35, p.195-207, 1957. apud HILL, J. C. G., An introduction to chemical engineering kinetics \& reactor design, New York: John Wiley \& Sons, 1977.

DITTMEYER, R.; HÖLLEIN, V.; DAUB, K., Membrane reactors for hydrogenation and dehydrogenation processes based on supported palladium, Journal of Molecular Catalysis A: Chemical, v.173, n.1-2, p.135-184, 2001.

HAUSEN, H. Neue Gleichungen für die Warmeübertragung bei freier oder erzwungener Strömung. Allg. Waermetech, v.9, p.75-79, 1959 apud PERRY, R.H.; GREEN, D.W. Perry's Chemical Engineers' Handbook, 7th ed. New York: McGraw-Hill, 1997.

HERMANN, C.; QUICKER, P.; DITTMEYER, R. Mathematical simulation of catalytic dehydrogenation of ethylbenzene to styrene in a composite palladium membrane reactor. Journal of Membrane Science.n.136, p.161-172, 1997.

KOC, R.; KAZANTIS, N. K.; MA, Y. H. A process dynamic modeling and control framework for performance assessment of $\mathrm{Pd} /$ alloy-based membrane reactors used in hydrogen production. International Journal of Hydrogen Energy, v.36, p.4934-4951, 2011.

KUMAR, S.; SHANKAR, S.; SHAH, P. R.; KUMAR, S.A Comprehensive Model for Catalytic Membrane Reactor. International Journal of Chemical Reactor Engineering, v.4, A5, p.1-27, 2006.

QUICKER, P.; HöLLEIN, V.; DITTMEYER, R. Catalytic dehydrogenation of hydrocarbons in palladium composite membrane reactors. Catalysis Today, v56, p.21-34, 2000.

SEBORG, D.E.; EDGAR, T.F.; MELLICHAMP, D.A. Process dynamics and control. $2^{\text {nd }}$ ed. John Wiley and Sons, 2004.

STANKIEWICZ, A. I.; MOULIJN, J. A. Process Intensification: Transforming Chemical Engineering. Chemical Engineering Progress, v. 96, p. 22-33, 2000.

STRATHMANN, H. Membrane Separation Processes: Current Relevance and Future Opportunities. AIChE Journal, v.47, n.5, p.1077-1087, 2001. 